\title{
Nonlinear waves of Bose-Einstein condensates in rotating ring-lattice potentials
}

\author{
A. Muñoz Mateo, ${ }^{1,2,3}$ V. Delgado, ${ }^{4}$ M. Guilleumas, ${ }^{3,5}$ R. Mayol,,${ }^{3,5}$ and J. Brand ${ }^{1,2}$ \\ ${ }^{1}$ Centre for Theoretical Chemistry and Physics, New Zealand Institute for Advanced Study, Massey University, Private Bag 102904 NSMC, \\ Auckland 0745, New Zealand \\ ${ }^{2}$ Dodd-Walls Centre for Photonic and Quantum Technologies, PO Box 56, Dunedin 9054, New Zealand \\ ${ }^{3}$ Departament de Física Quàntica i Astrofísica, Universitat de Barcelona, Martí i Franquès 1, E-08028 Barcelona, Spain \\ ${ }^{4}$ Departamento de Física, Facultad de Ciencias, Universidad de La Laguna, E-38200 La Laguna, Tenerife, Spain \\ ${ }^{5}$ Institut de Ciències del Cosmos, Universitat de Barcelona, ICC-UB, E-08028 Barcelona, Spain
}

(Received 20 November 2018; published 28 February 2019)

\begin{abstract}
We analyze the dynamics of Bose-Einstein condensates loaded in rotating ring lattices made of a few sites, and show how rotation maps the states found in this finite system onto those belonging to a static infinite lattice. Ring currents and soliton states in the absence of a lattice find their continuation in the presence of the lattice as nonlinear Bloch waves and soliton-like states connecting them. Both bright gap solitons and dark-soliton trains are shown to connect continuously to linear solutions. The existence of adiabatic paths upon varying rotation frequency between states with quantized supercurrents suggests highly controllable methods for the experimental generation of persistent currents.
\end{abstract}

DOI: 10.1103/PhysRevA.99.023630

\section{INTRODUCTION}

The experimental achievement of preparing, manipulating, and probing ultracold gases in periodic potentials has made it possible to observe elusive phenomena that typically belong to the realm of solid state physics. Ultracold atoms can quantum-simulate strongly interacting many-body systems [1] as well as dynamical processes in nonlinear media [2]. In the regime of large particle number per lattice site, the superfluid dynamics exhibited by Bose-Einstein condensates (BECs) loaded in (approximately) periodic potentials created by optical interference phenomena (optical lattices) provides an excellent sample of nonlinear phenomena. These range from the realization of atomic Bloch waves [3] that mimic the motion of electrons in metals to the generation of solitonic structures [4], which are the atomic equivalent of the nonlinear pulses currently used to carry information through optical fibers [5].

The early studies of BECs in periodic potentials were mainly focused on linear lattices, including one- (1D), two(2D), and three-dimensional (3D) lattices [3,6-8]. In these settings, the superfluid flow has been demonstrated to support many types of nonlinear waves [9-11]. The stability of these is intimately connected with the possibility for breakdown of superfluid flow and has been studied extensively $[6,12-14]$. Nonlinear waves in rotating 2D linear lattices (see, e.g., [15] and references therein) have been also studied. In the last years, with the advent of new experimental techniques capable of imprinting lattices along a ring [16-18], there has been an increasing interest in these configurations. They fulfill the Born-von Kármán boundary conditions that are presupposed in modeling crystals [19]. Most of the previous theoretical studies on this subject have started from discrete lattice models (see Refs. [20-27] and references therein), while nonlinear waves in continuous models with periodic potentials has received comparatively less attention [28-32].
The ring geometry introduces interesting features on its own, mostly because of its suitability for the study of metastable persistent currents $[33,34]$. In spite of the fact that persistent currents can also be found at a microscopic scale in normal metals $[35,36]$, metastable persistent currents are one of the hallmarks of superfluidity [37-40] and superconductivity $[41,42]$. Their generation and decay through phase-slip events in toroidal BECs stirred by optically induced weak links are currently the object of intensive research, since these systems have opened up a promising way of advancing towards the realization of atomtronic devices [43-46].

The present work aims to delve into the analysis of repulsively interacting BECs loaded in continuous ring lattices made of a small number of sites. By driving the lattice into angular rotation, the system exhibits the interplay of the rotating periodic potential and the intrinsic periodicity of the induced ring currents. In this situation, there also exists a relevant connection between angular quasimomentum and mean angular momentum. Previous analyses from nonlinear optics (e.g., the study of gap solitons in azimuthally modulated rings of Ref. [47]) have demonstrated that the ring lattice settings introduce significant changes in the properties of the associated nonlinear waves. However, such studies have only considered a particular type of nonlinear wave present in systems with a relatively large number of lattice sites, hence sharing many similarities with the limiting case of the infinite linear lattice. On the contrary, our approach starts by considering configurations containing a small number of wells $(1,2,3,4, \ldots)$ from the perspective of a periodic array. We study how the superfluid properties change as the number of wells and the nonlinear interaction strength are increased, approaching finally the well known limit of an infinite lattice [13]. This approach allows us to show in a unified and simple way the emergence of different types of nonlinear waves, namely Bloch waves, bright gap solitons, and dark solitons. 
Although these types of genuinely nonlinear states have been extensively studied in infinite lattices, they do not share a common theoretical framework and have so far been lacking a proper characterization in the case of finite, short lattices. Furthermore, we demonstrate the continuation of the nonlinear states into the noninteracting, linear regime, and discuss adiabatic pathways through parameter space to change the current and winding number, extending the work of Ref. [48], where the lattice potential was not considered, Ref. [49], where the effect of a symmetry-breaking potential (equivalent to a 1-site lattice) on the nucleation of ring currents was addressed, and Ref. [50], where nonlinear modes of rotating double-well rings have been studied. Our results contribute towards a cohesive picture of the physical phenomena in optical lattices.

\section{SYSTEM: THE RING LATTICE}

We consider a Bose-Einstein condensate confined to quasi$1 \mathrm{D}$ by a ring trap of mean radius $R$ at zero temperature and in the presence of a periodic potential along the azimuthal coordinate $\theta$ that rotates with angular frequency $\Omega$. By assuring that the number of particles per potential well is large enough for a mean-field model to be safely applied, the dynamics of the order parameter $\psi(\theta, t)$ in the corotating frame of reference follows the $1 \mathrm{D}$ Gross-Pitaevskii equation

$$
i \hbar \partial_{t} \psi=\left\{\frac{\left(-i \hbar \partial_{\theta}-m \Omega R^{2}\right)^{2}}{2 m R^{2}}+V(\theta)+g|\psi|^{2}\right\} \psi,
$$

where $V(\theta)=V_{0} \sin ^{2}(\pi R \theta / d)$ is the periodic potential introduced by an optical lattice with lattice constant $d$ and well depth $V_{0}$. The interparticle contact interaction has strength $g=2 \hbar^{2} a / m a_{\perp}^{2}$, determined by the $s$-wave scattering length $a$ and the size $a_{\perp}$ of a tight transverse confinement. The wave function $\psi$ is normalized to the number of particles in the ring $N=R \int d \theta|\psi|^{2}$.

The ring geometry provides a wave-number quantum $k_{R}=$ $1 / R$ imposed by the finite size of the system, so that the smallest amount of kinetic energy available in the ring is $E_{R} / 2$, where $E_{R}=\hbar^{2} k_{R}^{2} / m=\hbar \Omega_{R}$. This fact turns out to be crucial for the comparison with results obtained in the infinite lattice, where $E_{R}=0$. On the other hand, the periodic potential introduces the characteristic reciprocal lattice vector $k_{L}=$ $2 \pi / d$, which defines a maximum quasimomentum $\hbar k_{L} / 2$ at the end of the Brillouin zone and a corresponding lattice energy, or recoil energy, $E_{L}=\hbar^{2} \pi^{2} / 2 m d^{2}$. Both $k_{R}$ and $k_{L}$ are related by the commensurable ratio $M=k_{L} / k_{R}=2 \pi R / d$, which gives the number of lattice sites in the ring.

By writing Eq. (1) in the ring energy units $E_{R}$, and searching for the stationary states $\psi(\theta, t)=\psi(\theta) e^{-i \mu t / \hbar}$, with chemical potential $\mu$, we obtain a stationary nonlinear Schrödinger equation in dimensionless form

$$
\left\{\frac{1}{2}\left(-i \partial_{\theta}-\hat{\Omega}\right)^{2}+\hat{s} \sin ^{2}\left(\frac{M}{2} \theta\right)+\hat{g}|\hat{\psi}|^{2}-\hat{\mu}\right\} \hat{\psi}=0,
$$

where $\hat{\mu}=\mu / E_{R}, \hat{\Omega}=\Omega / \Omega_{R}, \hat{g}=2 a R / a_{\perp}^{2}$, the rescaled wave function is $\hat{\psi}=\sqrt{R} \psi$, and the lattice depth $V_{0}$ is measured relative to the ring energy $\hat{s}=V_{0} / E_{R}=s M^{2} / 8$, with $s=V_{0} / E_{L}$. It is worth noticing that $s$, instead of $\hat{s}$, is the usual parameter for identifying the dynamical regimes in the presence of the lattice, from the shallow lattice regime $(s \lesssim 1)$ up to the tight-binding limit $(s \gg 1)[9-11,14]$. For later use, we also define an average interaction parameter per lattice site $\eta=\hat{g} N / M$.

From Eq. (1), the local conservation of particle number in the rotating frame, $\partial_{\tau}|\hat{\psi}|^{2}+\partial_{\theta} \hat{J}=0$ (with $\tau=t \Omega_{R}$ ), determines the current density

$$
\hat{J}(\theta)=|\hat{\psi}(\theta)|^{2}[\hat{p}(\theta)-\hat{\Omega}],
$$

where $\hat{p}=\partial_{\theta} \arg (\psi)$ is the local canonical momentum. The superfluid velocity is given by $\hat{v}=\hat{J} /|\hat{\psi}|^{2}$. The mean angular momentum per particle $L_{z}$ (along the direction perpendicular to the ring) follows from

$$
L_{z}=\frac{\hbar}{N} \oint d \theta|\hat{\psi}(\theta)|^{2} \hat{p}(\theta) .
$$

The linear excitation modes $[u(x, t), v(x, t)]$ with energy $\hat{\omega}=\hbar \omega / E_{R}$ around the stationary state $\hat{\psi}$ fulfill the Bogoliubov equations [51]

$$
\begin{array}{r}
\left(H_{L}+2 \hat{g}|\hat{\psi}|^{2}\right) u+\hat{g} \hat{\psi}^{2} v=\hat{\omega} u, \\
-\left(H_{L}^{*}+2 \hat{g}|\hat{\psi}|^{2}\right) v-\hat{g}\left(\hat{\psi}^{*}\right)^{2} u=\hat{\omega} v,
\end{array}
$$

where $H_{L}=\left(-i \partial_{\theta}-\hat{\Omega}\right)^{2} / 2+\hat{s} \sin ^{2}(M \theta / 2)-\hat{\mu}$. The solutions to the Bogoliubov equations provide information about the linear dynamical stability in the ring lattice. In particular the presence of complex eigenfrequencies $\operatorname{Im}[\hat{\omega}] \neq 0$ indicates that the stationary state is dynamically unstable and prone to decay, since the associated unstable modes, if excited, grow exponentially $\propto \exp (\operatorname{Im}[\hat{\omega}] t)$.

It is instructive to start reviewing the eigenstates of the noninteracting $(g=0)$ ring lattice. At $\hat{\Omega}=0$, the stationary states have definite quasimomentum and play an equivalent role to that of winding number states, with definite angular momentum in the homogeneous ring. This equivalence still applies for nonzero $\hat{\Omega}$, by means of which the stationary states get their energies shifted inside the energy bands.

\section{A. Noninteracting regime}

According to the Bloch theorem, the eigenstates of the nonrotating system are the Bloch waves $\psi_{n, k}(R \theta)=$ $e^{i k R \theta} u_{n, k}(R \theta)$, where $\hbar k$ is the linear quasi- (or crystal) momentum that takes values within the first Brillouin zone $\hbar\left(-k_{L} / 2, k_{L} / 2\right]$. The function $u_{n, k}$ is periodic, and shares the period $d$ of the lattice [19], such that $u_{n, k}(R \theta)=u_{n, k}(R \theta+d)$, where $n$ is the band index, so that the Bloch waves are single valued in the ring, i.e., $\psi_{n, k}(R \theta)=\psi_{n, k}(R(\theta+2 \pi))$. Due to the finite size of the system, the quasimomentum takes discrete values $k=q k_{R}=q / R$, with $q=0, \pm 1, \ldots, M / 2$ being an integer number, and so the ring lattice bands exhibit a discrete structure.

From now on we will denote the Bloch waves by the two quantum numbers $|n, q\rangle \equiv \psi_{n, q}(\theta)=e^{i q \theta} u_{n, q}(\theta)$, or simply $|q\rangle$ if the band is implicitly assumed. There are just $M$ Bloch waves within the first Brillouin zone. In particular, when the lattice contains an even number of sites the crystal angular momentum reaches the edge of the Brillouin zone at $q=$ $M / 2$, while this limit is not reachable when the number of 


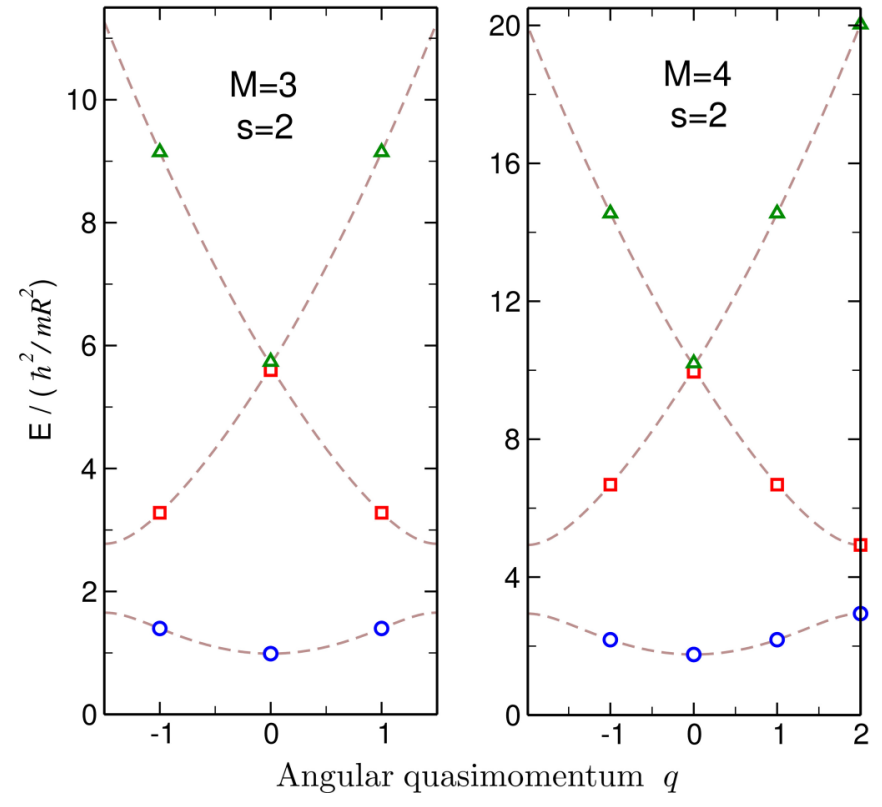

FIG. 1. Energy of the linear Bloch states (symbols) in the three lowest energy bands $n=1,2,3$ of nonrotating ring lattices with site numbers $M=3$ (left panel) and $M=4$ (right panel), sharing depth $s=V_{0} / E_{L}=2$ and radius $R$. The energy difference between the two systems is due to the different lattice spacing $d$. The continuous bands of the infinite lattices, with equal corresponding $d$, are represented by dashed lines.

sites is odd, and the maximum $|q|$ are $\pm q_{M}$, where $\left\{q_{M}=\right.$ $\lfloor M / 2\rfloor\}<M / 2$ is the maximum integer less than $M / 2$. For instance, let us consider two ring lattices, sharing radius $R$ and depth $V_{0}=2 E_{L}$, with 3 and 4 sites. The energy eigenvalues $E$ versus quasimomentum $q$ are represented in Fig. 1 (note that $E=\mu$ in this regime). There are three $(q=0, \pm 1)$ and four $(q=0, \pm 1,2)$ Bloch states per band, respectively. While for $M=4$ there is a single maximum-quasimomentum eigenstate $(q=2)$ at the band edge, for $M=3$ there are two (energy-degenerate) maximum-quasimomentum eigenstates $q= \pm q_{3}= \pm 1$ that belong to each band interior.

\section{B. Energy degeneracies}

As shown in Fig. 1, all the eigenstates in each energy band except those lying at the center $q=0$ and edge $q=M / 2$ of the Brillouin zone are doubly degenerate. As a consequence of this degeneracy, new eigenstates of nondefinite quasimomentum can be obtained by linear superposition of definite-quasimomentum Bloch waves; that is, $\psi=\alpha|n, q\rangle+$ $\beta|n,-q\rangle$, where $\alpha$ and $\beta$ are complex coefficients satisfying $|\alpha|^{2}+|\beta|^{2}=1$. Figure 2 highlights a representative case at $\hat{\Omega}=0$ inside the first energy band of a 4 -site lattice (open symbols). The two panels collect data from the numerical solution of Eq. (2) with $\hat{g}=0$. Whereas the degeneracy of states with quasimomenta $q=1$ and $q=-1$ is indicated by a single symbol in the energy graph (lower panel of Fig. 2), it corresponds to a continuum of states $\alpha|1\rangle+\beta|-1\rangle$ with mean angular momentum $L_{z} \sim \hbar\left(|\alpha|^{2}-|\beta|^{2}\right)$ in the interval $\hbar[-1,1]$ (represented by three connected symbols in the upper panel of Fig. 2). As we will see, all the states in the mentioned

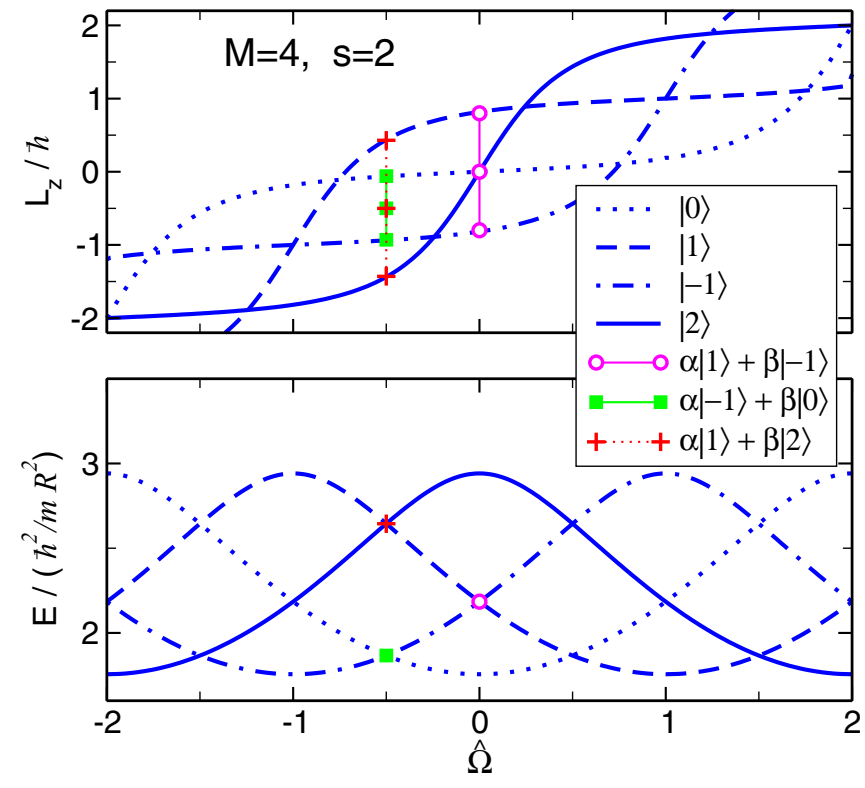

FIG. 2. Energy (lower panel) and mean angular momentum (upper panel) of linear Bloch states in the first energy band of a ring lattice rotating at rate $\hat{\Omega}=m R^{2} \Omega / \hbar$ and with the same parameters as in the right panel of Fig. 1. The symbols, overlapped in the energy graph and connected by thin vertical lines in the angular momentum graph, indicate three particular cases of degenerate states.

interval find continuation in the nonlinear regime. The states with definite quasimomentum $q$ become the nonlinear Bloch waves, whereas those without definite $q$ transform into solitonic states.

The linear superpositions of degenerate Bloch waves give rise to new symmetries that do not share the usual periodic features of the Bloch states. As an example, taken from the ring lattice with $M=3$ of Fig. 1, the linear combinations of the degenerate eigenstates $\psi_{1,1} \equiv|1,1\rangle$ and $\psi_{1,-1} \equiv|1,-1\rangle$ give rise to states with one or two density peaks, for the symmetric $|1,1\rangle+|1,-1\rangle$ and the antisymmetric $|1,1\rangle-$ $|1,-1\rangle$ cases, respectively (see Fig. 6 for the continuation of these states in the nonlinear regime). As we will show below, these two different symmetries, having zero mean angular momentum, are manifested in two separated families of nonlinear, soliton-like states. Furthermore, for other linear combinations of Bloch waves (other than the symmetric and the antisymmetric in this case) with nonzero mean angular momentum, it is not possible to find an equivalent nonlinear continuation by keeping $\hat{\Omega}=0$. Such nonlinear continuation does still exist for nonzero rotation rate $\hat{\Omega} \neq 0$.

\section{Rotation}

Additional energy degeneracies are induced by rotation. To see this, we first notice that the few-site rotating lattice maps onto the infinite lattice due to the fact that the Gross-Pitaevskii equation for the Bloch-wave amplitude $\hat{u}_{n, q}(\theta)$ in the rotating frame,

$$
\left\{\frac{1}{2}\left(-i \partial_{\theta}+q-\hat{\Omega}\right)^{2}+\hat{V}(\theta)+\hat{g}\left|\hat{u}_{n, q}\right|^{2}-\hat{\mu}\right\} \hat{u}_{n, q}=0,
$$


is also the equation of motion of a Bloch state with quasimomentum $q^{\prime}=q-\hat{\Omega}$ in the static infinite lattice. In particular, in the noninteracting linear regime $(\hat{g} \rightarrow 0)$ the energy $E_{q}(\hat{\Omega})$ (in units of $E_{R}$ ) of a Bloch wave of (discrete) quasimomentum $q$ in the rotating ring lattice coincides with the energy $E^{\infty}(q-$ $\hat{\Omega}$ ) of a Bloch wave of quasimomentum $q^{\prime}=q-\hat{\Omega}$ in the static infinite lattice. Therefore, for fixed $q$, by varying conveniently the parameter $\hat{\Omega}$ one can expand the eigenstates of the infinite lattice in the whole Brillouin zone $q^{\prime} \in(-\pi / d, \pi / d]$. Since the group velocity in the infinite lattice is given by $\hat{v}=\partial_{q^{\prime}} E^{\infty}\left(q^{\prime}\right)$ [19], the above equivalence enables us to infer the superfluid velocity in the rotating ring lattice as a function of the angular velocity:

$$
\hat{v}(\hat{\Omega})=-\partial_{\hat{\Omega}} E_{q}(\hat{\Omega}) .
$$

As $\hat{\Omega}$ varies, the Bloch states $|q\rangle$ (labeled by their quasimomentum $q$ at $\hat{\Omega}=0$ ) shift their positions in the energy graph (see lower panel of Fig. 2) according to the periodicity introduced by both the ring, $\Delta \hat{\Omega}=M$, and the lattice, $\Delta \hat{\Omega}=$ 1. Then, in the Brillouin zone $E_{q}(\hat{\Omega})=E_{q}(\hat{\Omega}+l M)$, with $l$ integer. Besides, consecutive Bloch waves $|q\rangle$ and $|q+1\rangle$ swap their energies after a $\Delta \hat{\Omega}=1$ interval in the angular rotation, such that $E_{q}(\hat{\Omega}=q+1)=E_{q+1}(\hat{\Omega}=q)$. In general, for each pair of Bloch waves with $\left(q_{1}, q_{2}\right)$, these symmetries imply energy degeneracies at $\hat{\Omega}=\left(q_{1}+q_{2}\right) / 2$ and $\hat{\Omega}=\left(q_{1}+q_{2}+M\right) / 2$, that is, at either integer or half-integer $\hat{\Omega}$. The total number of degeneracies per energy band is the number of combinations $\left(\begin{array}{c}M \\ 2\end{array}\right)$. Two examples are explicitly indicated in Fig. 2. Symbols at $\hat{\Omega}=-0.5$ mark the superpositions $\alpha|-1\rangle+\beta|0\rangle$ and $\alpha|1\rangle+\beta|2\rangle$. Again, the degenerate states expand continuum intervals in the graph of the mean angular momentum per particle (upper panel of Fig. 2), with $L_{z} \sim \hbar[-1,0]$ and $L_{z} \sim \hbar[-1.5,0.5]$, respectively.

Taking into account Eq. (3), the quantization of the circulation around the ring lattice in units of the "flux quantum" $\phi_{0}=2 \pi \hbar / m$ takes the form

$$
\hat{\Gamma}=\frac{1}{2 \pi} \oint d \theta \hat{p}(\theta)=\frac{1}{2 \pi} \oint d \theta(\hat{v}+\hat{\Omega})=l,
$$

with $l=0,1,2 \ldots$ This expression is clearly equivalent to the well known fluxoid quantization in superconductivity [52]. It amounts to encircling $l$ vortices inside the ring, for the phase jumps in $2 \pi l$ when encircling the system. To better understand the reference to vortices in this context, it is useful to consider an equivalent 3D toroidal system provided with an effective 1D geometry by means of a tight transverse confinement. Then, quantized persistent currents in the 1D ring (as a zero-width limit of the 3D torus) correspond to 3D flows generated by vortices located at the center of the real toroidal system. Singular situations arise if a vortex is located exactly on the ring, producing a node in the corresponding wave function. This occurs at a particular value of the rotation rate in the transit of a vortex from the outer to the inner regions of the ring or vice versa. Since each vortex (i.e., each node) on the ring imposes a sudden $\pi$-phase jump at its location, the phase winding around the ring now leads to

$$
\frac{1}{2 \pi} \mathrm{P} \oint d \theta \hat{p}(\theta)=\frac{1}{2 \pi} \mathrm{P} \oint d \theta(\hat{v}+\hat{\Omega})=\frac{\nu}{2},
$$

where $v$ is the number of nodes and $\mathrm{P}$ denotes the Cauchy principal value. Thus the presence of nodes in the wave function extends the quantization of circulation Eq. (8) to half integers of $h / m$.

From Eq. (3), when the superfluid velocity vanishes, then $\hat{p}(\theta) \equiv \partial_{\theta} \arg (\psi)=\hat{\Omega}$ and hence, according to the quantization of circulation, the phase has to be linear, $\arg (\psi)=j \theta$, with $j$ being an integer or half integer. For a Bloch wave $|q\rangle$, from Eq. (7), the superfluid velocity vanishes at the minima and maxima of the dispersion relation $E_{q}(\hat{\Omega})$ in the rotating frame. The minimum corresponds to the ground state, at $\hat{\Omega}=q$ (see, for example, the lower panel of Fig. 2); it has no nodes and the linear phase takes the value $\arg (\psi)=q \theta$. Substituting these results in Eq. (4) and making use of the normalization condition one finds that at the minimum of the dispersion relation $E_{q}(\hat{\Omega})$ of a Bloch wave $|q\rangle$, which occurs at $\hat{\Omega}=q$, the mean angular momentum per particle takes the value $L_{z}=\hbar q$, corresponding to the presence of $q$ vortices inside the ring lattice.

For a Bloch wave $|q\rangle$, the superfluid velocity also vanishes at the energy maxima, on the edge of the Brillouin zone $\hat{\Omega}=q+M / 2$. In this case the phase reads $\arg (\psi)=(q+$ $M / 2) \theta$, indicating the presence of $M$ nodes on the ring. The mean angular momentum per particle takes correspondingly half-integer (for $M$ odd) or integer (for $M$ even) values of $\hbar$ (see, e.g., Fig. 2). In the absence of rotation, the single-value property of the wave functions precludes the fulfillment of Eq. (9) for odd number of nodes.

The above results lead to the following picture for a rotating ring lattice with $M$ sites (see, e.g., Fig. 2). Starting from the ground state of a Bloch wave $|q\rangle$ in the first energy band (which occurs at $\hat{\Omega}=q$ and has a mean angular momentum per particle $L_{z}=\hbar q$ ), as $\hat{\Omega}$ increases adiabatically so does the condensate energy $E_{q}(\hat{\Omega})$ while $L_{z}$ remains almost unaltered reflecting the superfluidity of the system. This situation holds until $E_{q}(\hat{\Omega})$ approaches a maximum, which occurs at $\hat{\Omega}=$ $q+M / 2$ and corresponds to the presence of $M$ vortices lying exactly on the ring lattice. At this point $L_{z}$, which increases suddenly in the vicinity of this configuration, takes the value $L_{z}=q+M / 2$. Finally, as $\hat{\Omega}$ increases further the above $M$ vortices enter the ring and $L_{z}$ keeps increasing up to $L_{z}=$ $q+M$. This picture essentially remains true in the nonlinear regime.

\section{NONLINEAR WAVES}

A simple picture, based on the continuation of states from the noninteracting regime, allows for the generation of the different families of nonlinear waves in the ring lattice. Figure 3 shows the outcome of this approach for the lattices considered in Fig. 1. Families of states proceeding from the first (solid lines) and second (dashed lines) energy bands are shown at $\hat{\Omega}=0$. Gap solitons have their origin at the mentioned energy degeneracies of the linear regime. This is the case for the families originating from the combinations $|1,1\rangle \pm|1,-1\rangle$ for $M=3$ (top panel of Fig. 3). For $M=4$ (lower panel) the linear origin of the gap-soliton family is not apparent. It bifurcates from the top of the first energy band in the nonlinear regime. However, it can be tracked back up to the linear 

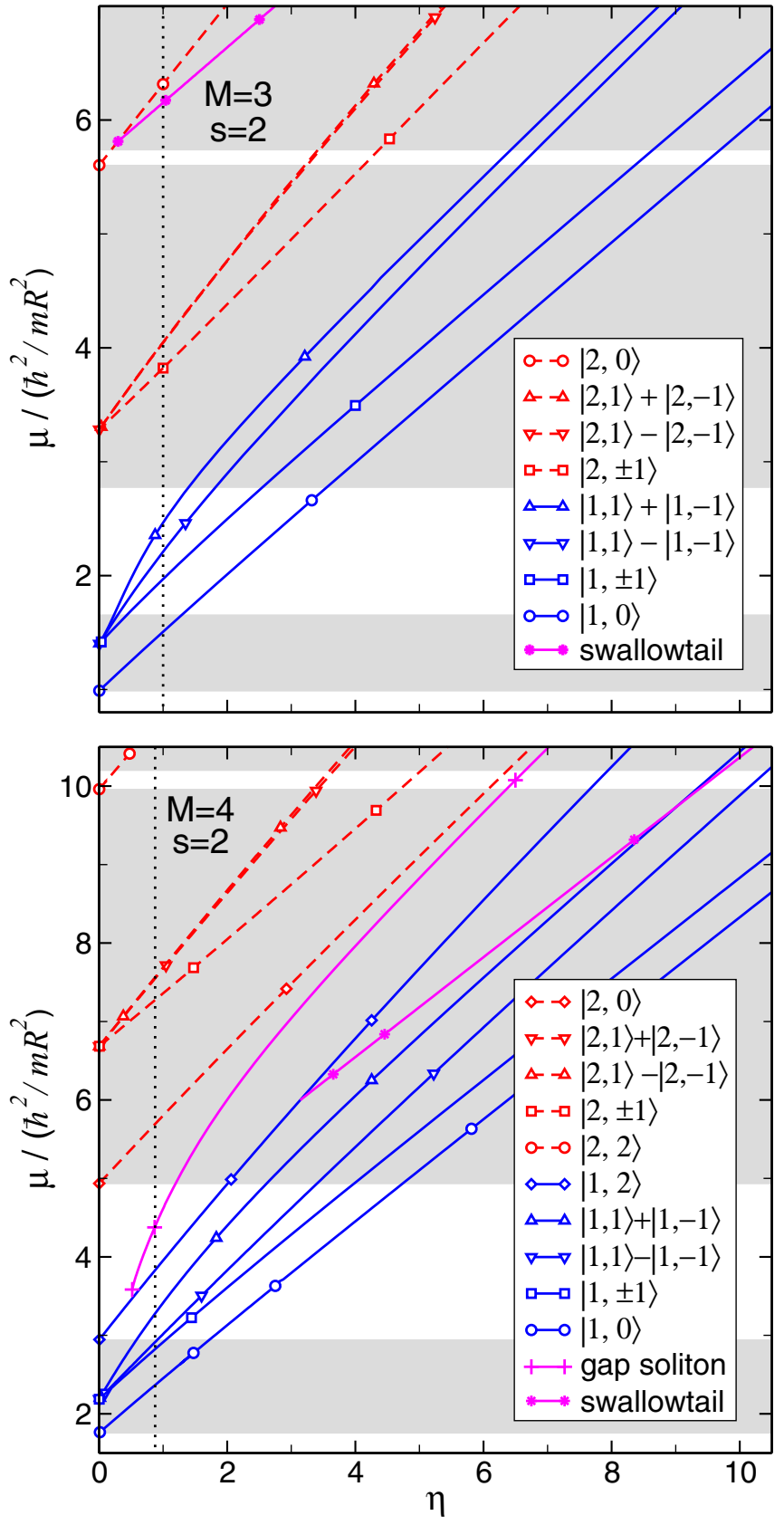

FIG. 3. Trajectories in the chemical potential versus interaction $(\eta=\hat{g} N / M)$ graph of nonlinear states living in the ring lattices of Fig. 1 at $\hat{\Omega}=0$. The gray-shaded regions represent the linear energy bands of the infinite lattices. The vertical dotted lines indicate particular values of the nonlinearity $(\eta \sim 1)$ for which the state density profiles are shown in Fig. 6.

regime at $\hat{\Omega}=0.5$, where it originates from the combinations $|1,2\rangle \pm|1,1\rangle$.

In general, an increasing interaction strength leads the nonlinear states to resonate with excitation modes associated with the underlying linear energy spectrum. These resonances are responsible for the generation of pitchfork bifurcations that translate into swallowtails at the maxima of the nonlinear energy bands (shown in Fig. 3, at the second band for $M=3$ and at the first band for $M=4$ ), or saddle node bifurcations

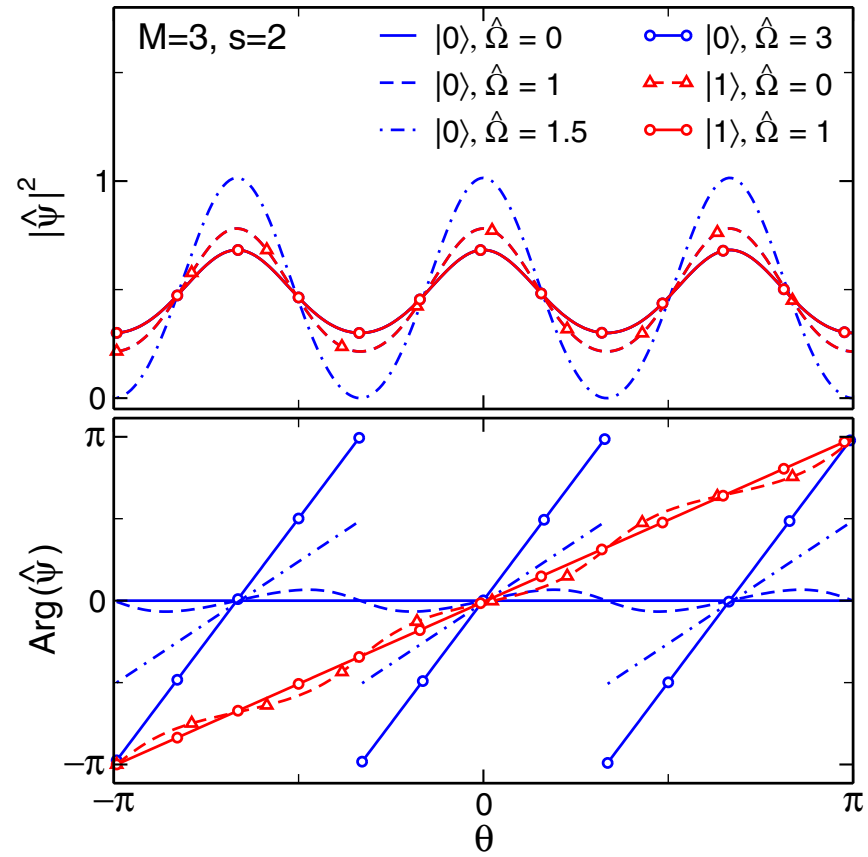

FIG. 4. Number density and phase profiles of the nonlinear Bloch waves $|0\rangle \equiv|1,0\rangle$ and $|1\rangle \equiv|1,1\rangle$ in the $M=3$ ring lattice of Fig. 1 for different rotation values $\hat{\Omega}$. The phase winding induced by rotation causes $|0\rangle$ to evolve from a noncurrent state at $\hat{\Omega}=0$, through a soliton-train-like state at $\hat{\Omega}=1.5$, into a vortex-like (ringcurrent) state at $\hat{\Omega}=3$. Several density profiles are equal, having overlapped curves in the top panel: on the one hand, the three states $|0\rangle$ at $\hat{\Omega}=0,|0\rangle$ at $\hat{\Omega}=3$, and $|1\rangle$ at $\hat{\Omega}=1$; on the other hand, the two states $|0\rangle$ at $\hat{\Omega}=1$ and $|1\rangle$ at $\hat{\Omega}=0$.

of gap solitons in higher energy gaps. In what follows, we provide evidence for these statements.

\section{A. Persistent current switch}

The nonlinear families of Bloch waves support supercurrents that can be controlled by means of rotation. In Fig. 4, we have summarized the changing configuration (density and phase) of two Bloch waves, $|0\rangle \equiv|1,0\rangle$ and $|1\rangle \equiv|1,1\rangle$, with varying angular rotation. The system parameters are the same as in the 3-site lattice of Fig. 1, but now with an interaction parameter $\eta=1$. The energy and mean angular momentum of these Bloch waves can be also compared with other stationary states in Fig. 5. Monitoring the phase of state $|0\rangle$ in the transit from $\hat{\Omega}=0$ to $\hat{\Omega}=3$ (lower panel Fig. 4), one can see that three nodes make simultaneously their appearance at $\hat{\Omega}=1.5$ (dot-dashed lines), just when the state reaches its maximum chemical potential as a function of $\hat{\Omega}$ (see Fig. 5). At this point the circulation due to the presence of three nodes is $\hat{\Gamma}=$ $3 / 2$. In the transit from $\hat{\Omega}<1.5$ to $\hat{\Omega}>1.5$ the circulation jumps from $\hat{\Gamma}=0$ to $\hat{\Gamma}=3$, indicating the entry of three vortices inside the ring. The mean angular momentum keeps increasing with $\hat{\Omega}$ from $L_{z}=0$ at $\hat{\Omega}=0$ up to $L_{z}=3 \hbar$ at $\hat{\Omega}=$ 3 , where the density profile matches the original configuration at $\hat{\Omega}=0$.

The Bloch state $|1\rangle$ makes an equivalent transit from $\hat{\Omega}=1$ to $\hat{\Omega}=4$. The density profiles are exactly the same as those 


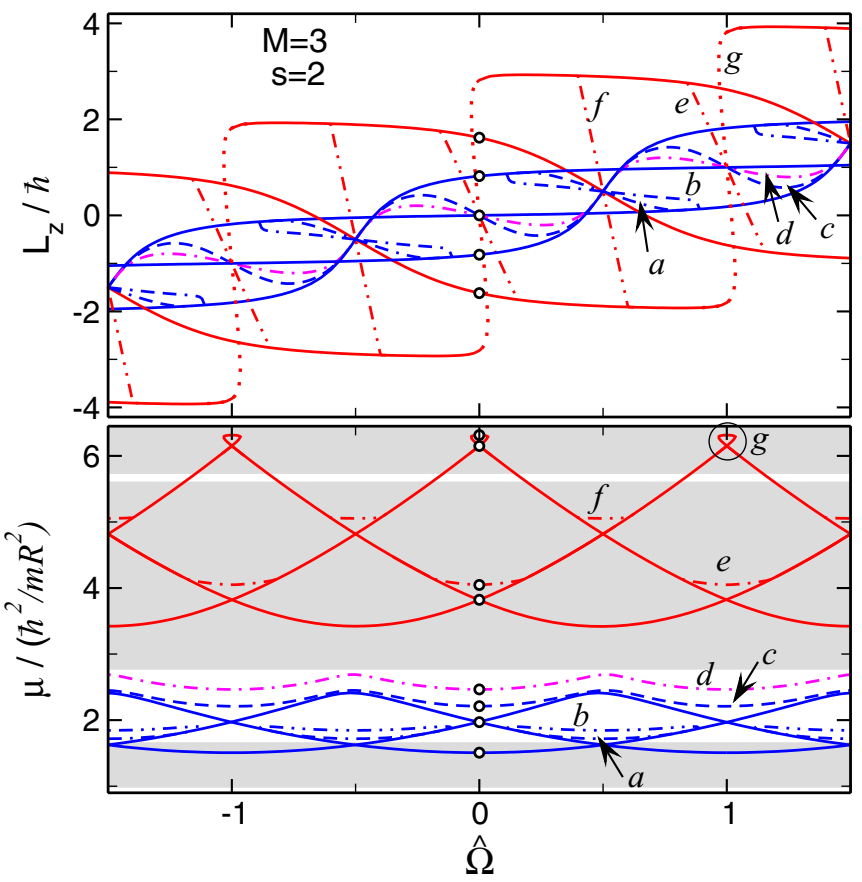

FIG. 5. Mean angular momentum per particle (top panel) and chemical potential (bottom panel) of stationary states in the first and second energy bands of a rotating ring lattice with $M=3, s=2$, and $\eta=1$. Open symbols indicate the nonrotating eigenstates depicted in the left panels of Fig. 6. The shaded regions correspond to the linear energy bands, and the solid lines represent the Bloch states. The discontinuous lines correspond to nonlinear states without definite quasimomentum. For instance, states of the families labeled by $a$ and $b$, in the first band, and $e$ and $f$, in the second band, form intraband swallowtails. The families $c$ and $d$ are made of gap-solitonlike states, whereas the (encircled) states $g$ in the second energy band form out-of-band swallowtails whose upper part is associated with the dotted lines in the angular momentum panel.

of $|0\rangle$ at one less unit of $\hat{\Omega}$. The phase profiles of state $|1\rangle$, instead, reflect one additional unit of circulation. The mean angular momentum increases from $L_{z}=\hbar$ to $L_{z}=4 \hbar$.

This smooth variation of Bloch waves suggests an experimental procedure to switch between persistent currents in toroidal systems by means of ring lattices. The advantage with respect to previous methods using Gaussian weak links to drag a BEC [45] is the absence of sudden, uncontrolled phase slips. By using an $M$-site lattice, persistent currents that differ in $M$ winding numbers can be smoothly switched. Nevertheless, it is important to add that the Bogoliubov analysis of the states making such connections reveals dynamical instabilities for intermediate values of $\hat{\Omega}$. For instance, in the particular case shown in Fig. 4, and within the transit of $|0\rangle$ in the range $\hat{\Omega} \in[0,3]$, the intermediate states in the window $\hat{\Omega} \in$ $[0.92,2.08]$ are unstable. However, a smooth transit, faster than the typical growth rate of the unstable modes, through these intermediate regions could still adiabatically (passing through all the intermediate states of the Bloch wave) lead the system into the final stable current states. This transition is not qualitatively different from the driven transition of a linear system in the observation of Bloch oscillations.
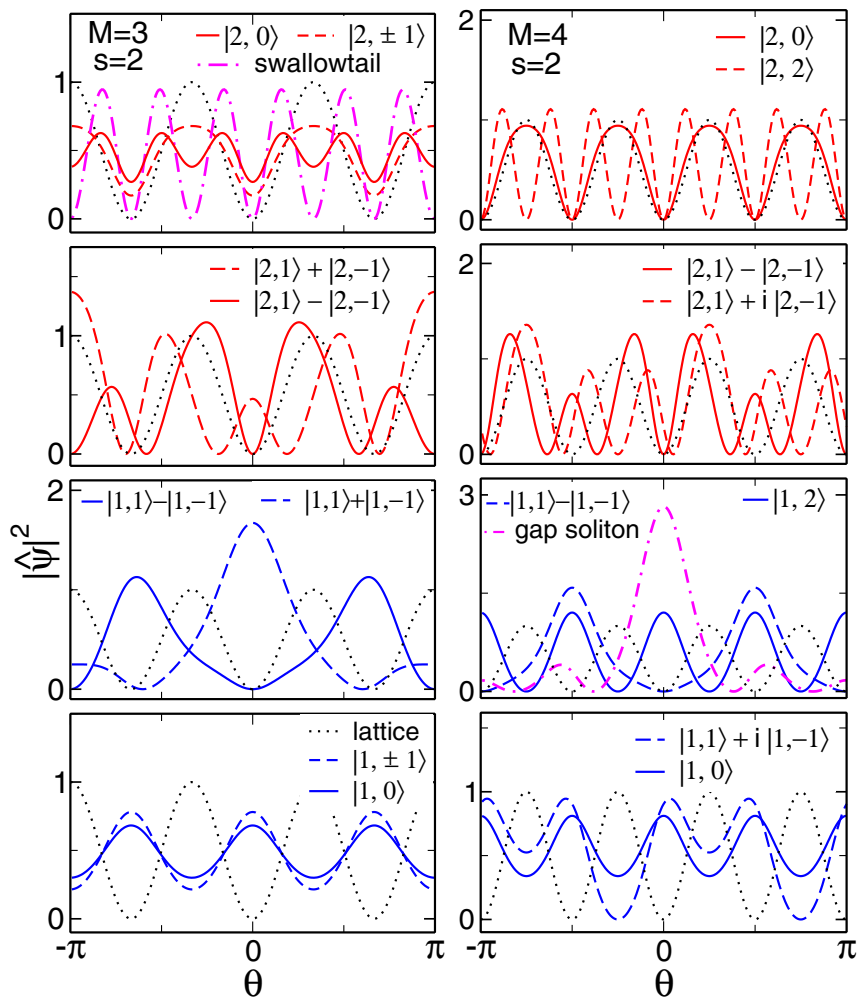

FIG. 6. Azimuthal densities of the stationary states of the GrossPitaevskii Eq. (2) with $\hat{\Omega}=0$ in the ring lattices of Fig. 1 and nonlinearity given by the vertical dotted lines in Fig. 3 . The dotted lines represent the lattice profile.

\section{B. Dark-soliton-like states}

The most striking difference in the energy bands with respect to the linear regime is the generation of swallowtails beyond a threshold value of the interaction strength $[28,30,31]$. These structures of the energy bands break the smooth connection described before between stable states supporting persistent currents. The swallowtails are shaped at the local maxima of the nonlinear energy bands, as can be seen (encircled) in the second band of the bottom panel of Fig. 5, and more clearly in both the first and second energy bands in Fig. 7. These structures, which will be referred as out-of-band swallowtails, are developed at the edges of the Brillouin zone in the lowest energy band when $g \bar{n}>s E_{L} / 2$, where $\bar{n}=N / 2 \pi R$ is the average density, or at the center of the Brillouin zone in the first excited (second) energy band when $g \bar{n}>4 E_{L}\left[\sqrt{1+(s / 8)^{2}}-1\right]$ (as it is the case in the bottom panel of Fig. 5). In the ring units, these thresholds are $\eta_{1}=\pi M s / 8$ and $\eta_{2}=\pi M\left[\sqrt{1+(s / 8)^{2}}-1\right]$, respectively, with $\eta_{2}<\eta_{1}$.

Along with the out-of-band swallowtails, there are also swallowtails in the energy-band interior, as those shaped by the curves $a, b, e$, and $f$ in Fig. 5. These structures, which we will refer to as intraband swallowtails, are made of periodic states that have different period $\lambda d$ ( $\lambda$ integer) from the lattice [53]. They have been interpreted as states made of dark solitons, due to the presence of nodes in their wave functions (as can be seen in Fig. 6). As we demonstrate below, these 
families of nonlinear waves proceed from the combinations of linear degenerate states.

\section{Out-of-band swallowtails and sudden phase slips}

Let us first investigate the ring rotating "lattice" with just one site $M=1$. For weak periodic potentials, this minimal system can be seen as a toroidal condensate in the presence of a wide weak link. As a result the associated physics of persistent currents in a ring can be recovered [46]. Specifically, the case of a weak sinusoidal potential in a ring has been addressed from the point of view of the generation and stability of dark-soliton states [49].

In Fig. 7 we show the chemical potential and the mean angular velocity of the stationary states with $s=2$ and interaction $\eta=2$. Since in this case $\eta_{1}=0.79$ and $\eta_{2}=0.1$, there are swallowtails at the first and the second energy bands. As a consequence, the ground state $|1,0\rangle$ at $\hat{\Omega}=0$, indicated by the label A in the bottom panel of Fig. 7, cannot smoothly transit from $\hat{\Omega}=0$, where it has $L_{z}=0$, up to $\hat{\Omega}=1$, where it has $L_{z}=\hbar$ (point $\mathrm{A}^{\prime}$ ). The energy curve of state $|1,0\rangle$ is broken into equal pieces with different circulation centered around the integer values of $\hat{\Omega}$. Each piece represents a metastable supercurrent state that effectively stretches beyond the edge of the Brillouin zone, at half-integer values of $\hat{\Omega}$, where it is not the ground state. Eventually the energy path reaches a maximum at a critical rotation rate $\hat{\Omega}_{c}$ (point $\mathrm{T}$ ), beyond which it does not exist. As a result, the adiabatic evolution through the swallowtail is not possible, and just changing $\Omega$ beyond the outer edge of the tail structure results in nonadiabatic dynamics, a sudden phase slip, which will also change the total circulation by $2 \pi$. The path from A to T in Fig. 7 can be done adiabatically, but increasing $\Omega$ any further will cause the system to drop to the line connected to $\mathrm{A}^{\prime}$ (plus phononlike excitations). A similar dynamics takes place in the absence of lattice, as has been demonstrated in toroidal systems with a weak link $[45,46]$, where an increase of rotation beyond the swallowtail $\hat{\Omega}>\hat{\Omega}_{c}$ brings a metastable state through a sudden phase-slip event, which changes the circulation, into the corresponding ground state.

The crossing energy paths of two (consecutive) metastable supercurrents are connected, at their maxima, by a family of solitonic states. The state $\mathrm{B}$ at $\hat{\Omega}=0.5$ (see Fig. 7) represents a clear example of the latter. The energy curves of these families trace the out-of-band swallowtails, which reflect the presence of hysteresis in the system [32,45]. It is interesting to compare the states living on the swallowtails, e.g., points $\mathrm{B}$ and $\mathrm{B} 1$ in Fig. 7, with the corresponding states on the next energy band for the same rotation rate $\hat{\Omega}$, i.e., states $\mathrm{C}$ and $\mathrm{C} 1$, respectively. They present the same number of nodes. While state $\mathrm{B}$ has one node at the maximum of the periodic potential, state $\mathrm{C}$ has one node at the minimum. Similarly in the second band, B1 presents two nodes, one of which lays at the potential maximum, whereas $\mathrm{C} 1$ has both nodes within the potential well. As noted in Ref. [49], this difference is crucial for the dynamical stability of the states, in such a way that those states having nodes at the maxima are unstable, but those with nodes inside the potential well are dynamically stable.

The out-of-band swallowtails are the result of a pitchfork bifurcation of the family of nonlinear Bloch waves situated at
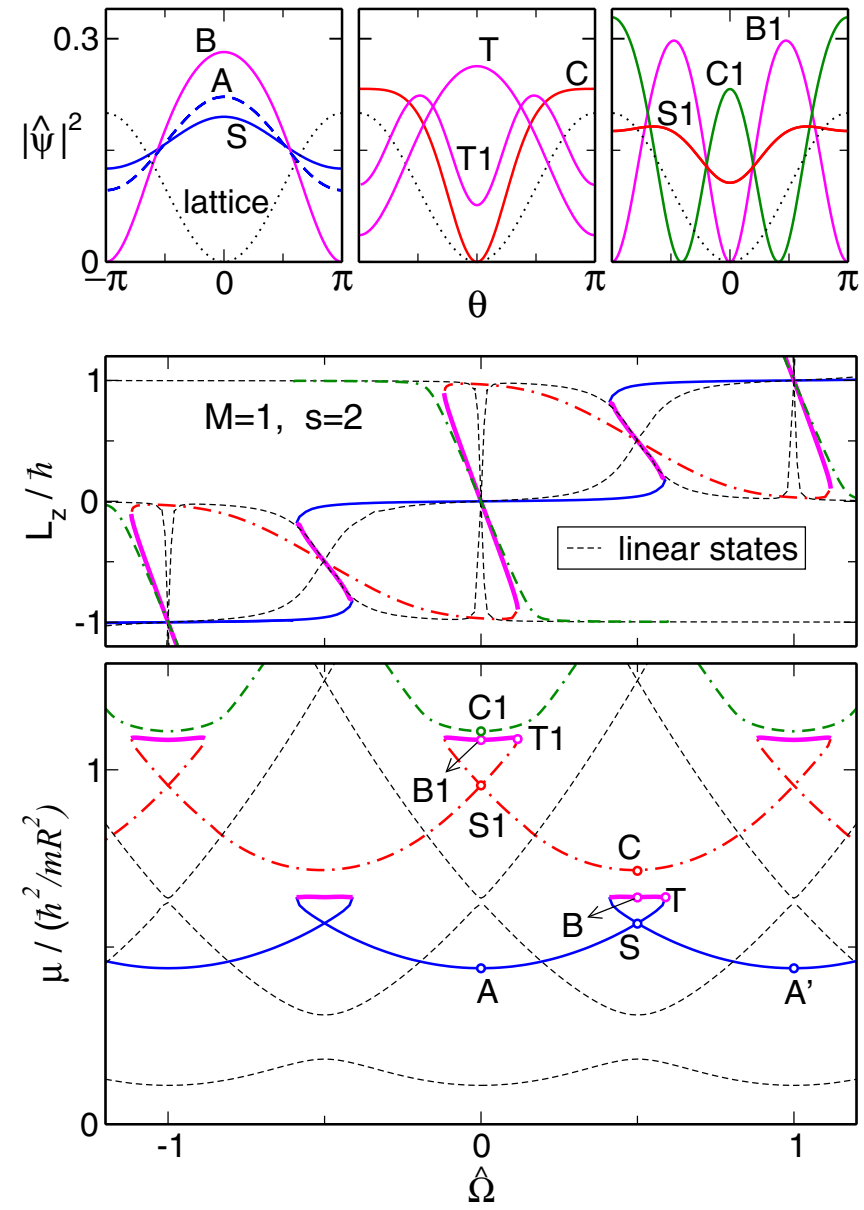

FIG. 7. Stationary states of the Gross-Pitaevskii Eq. (2) with interaction parameter $\eta=2$ for the first energy bands of a rotating ring lattice with $M=1$ and $s=V_{0} / E_{L}=2$. Top panels: Density profiles of labeled states. Lower panels: Chemical potential (bottom) and mean angular momentum per particle (middle) in the first (continuous thin lines), second (dash-dotted lines), and third (double-dash-dotted lines) energy bands. Families of soliton-like states (continuous thick lines) form the out-of-band swallowtails in the first and second energy bands.

the upper edge of the energy bands. To illustrate this point, we make use of a system with $M=4, s=2, \hat{\Omega}=0$, and varying interaction $\eta$, where swallowtails emerge in the first energy band at $\eta_{1}=\pi$. Figure 8 shows the excitation modes (dots) of the family of states $|1,2\rangle$ (thick continuous and dashed lines), which originates at the top of the first (linear) energy band. The excitation modes have also continuation from the linear regime, where they coincide with the eigenstates of the linear Hamiltonian. As maxima of the energy band, the states $|1,2\rangle$ have zero angular momentum (see the top panel of Fig. 8). However, at $\eta=\eta_{1}$ (indicated by arrows in the graphs) two new, degenerate states with opposite currents bifurcate (thin continuous and dash-dotted lines) due to the resonance with an excitation mode that originates from the second energy band (see the middle panel of Fig. 8).

For higher interaction strength $\eta>\eta_{1}$ the family $|1,2\rangle$ includes new excitation modes with purely imaginary frequencies (bottom panel of Fig. 8), denoting its dynamical 


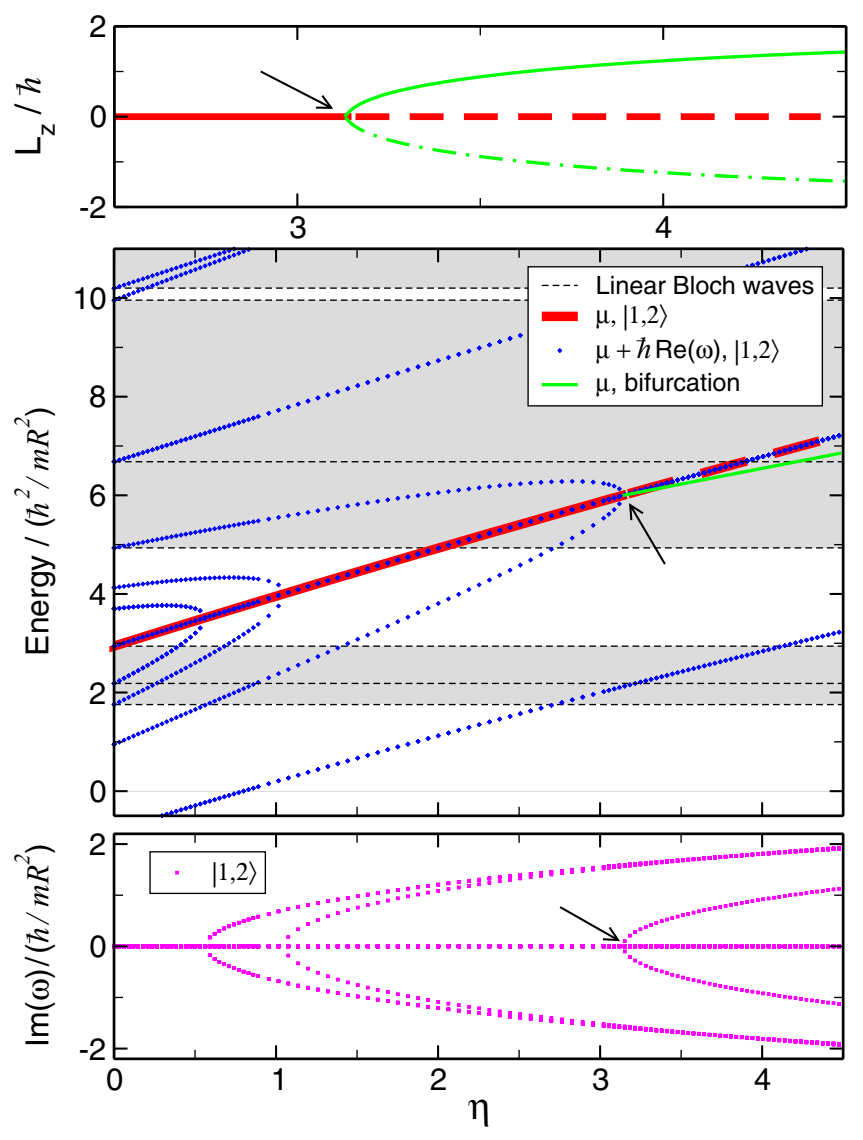

FIG. 8. Top panel: Pitchfork bifurcation (marked by the arrow) of states $|1,2\rangle$ (thick lines) generating an out-of-band swallowtail in the first energy band of a ring lattice with $M=4, s=2$, and $\hat{\Omega}=0$. Two new current states with the same chemical potential (thin lines) emerge at interaction $\eta_{1}=\pi$. Middle panel: Chemical potential of states $|1,2\rangle$ (continuous and thick dashed lines) and, superimposed, sum of chemical potential plus real part of Bogoliubov excitation energies (symbols) as a function of the interaction. Bottom panel: Imaginary part of the frequencies of Bogoliubov excitations as a function of interaction.

instability. The bifurcating current-carrying states inherit the stability properties of the parent state before the bifurcation. In the present case they are also dynamically unstable due to resonances that take place at lower interaction strength $\eta<\eta_{1}$ (see lower panels of Fig. 8) with excitation modes proceeding from the first linear energy band.

\section{Intraband swallowtails}

For $M>1$, additional swallowtails originate from the connection between two crossing paths (in the $\mu-\hat{\Omega}$ graph) of nonlinear Bloch waves having different quasimomentum. The system shown in Fig. 5, a ring lattice with $M=3, s=2$, and $\eta=1$, shows multiple examples of these structures along with small out-of-band swallowtails in the second energy band. Contrary to the latter, the intraband swallowtails exist for arbitrarily small values of the interaction and originate from the energy degeneracies in the noninteracting regime. They clearly resemble the solitonic curves between winding number states found in the absence of a lattice [48]. But unlike such case, there are two families of solitonic states (and not only one, although hard to distinguish at the scale of Fig. 5) making the connection between states with definite quasimomentum on the upper part of each crossing. The number of these (double) swallowtails, per energy band, equals the number of energy degeneracies (or crossings) between pairs of the $M$ Bloch waves.

This scenario is a generalization of the particular cases that have been presented in the literature [53]. In order to make the connection between the two states of different quasimomentum, a number of nodes matching the difference in the circulation $\Delta \hat{\Gamma}$ are generated on the ring. The nodes appear in the states situated just at the middle of the swallowtails, and evolve into density depletions that become shallower as the solitonic states approach the merging point with the Bloch states. The presence of the nodes breaks the symmetric pattern of the lattice and gives rise to new periods.

A remark about the solitonic character of the states living on the described swallowtails is in order. Although a general classification of these states as trains of dark solitons can be done, as regarding the presence of nodes, they can also be considered trains of bright solitons, as regarding the localization of the particle density. The next subsection clarifies this point.

\section{Bright solitons}

The allusion to bright solitons within the few-site lattice needs additional remarks, since the clear signature of a gap soliton, the localization, can only be manifested in a long lattice (compared with the spacing $d$ ). Once a bright soliton candidate has been found in a small lattice with $M_{0}>1$, we look at equivalent states in longer lattices by keeping the spacing $d$ constant and increasing the number of sites $M$. In doing so the ring radius is increased, and the kineticenergy quantum is reduced, so that the interaction parameter $\eta \propto R / M$ does not change. Additionally, to follow the transit towards the infinite-lattice limit, we have to keep the parity in the number of sites so that the group velocity $\hat{v}=\partial_{q} E(q)$, and then the relative position of the state in the energy band, does not change. This constrains the lattice series to number of sites $M=\left(M_{0}\right)^{j}, j=1,2,3, \ldots$ matching the powers of the original lattice $M_{0}$.

With this scheme, let us consider the family of gap solitons (born in the first energy gap) in a series of static ring lattices with $s=10$ and $M_{0}=2$, as shown in Fig. 9, for $M=2,4,8,16$ and $M=64$. The gap-soliton trajectories in the $\mu-N$ graph and also their configurations are practically indistinguishable within the energy gap, a fact that allows us to study these states in low- $M$ lattices. Only the approach to the second energy band produces relevant differences. For increasing $M$ the trajectories bend towards the lowest energy state in the second band. The bending increases with $M$ approaching the limit of an infinite lattice, where gap solitons cannot live inside the energy band. However, the finite size of the rings releases this constraint. As soon as the gap solitons enter the bands, long oscillating tails are developed that are responsible for the observed bending in their trajectories. Notwithstanding, a clear density peak remains as the gap soliton signature. In what follows, we elaborate on these issues. 


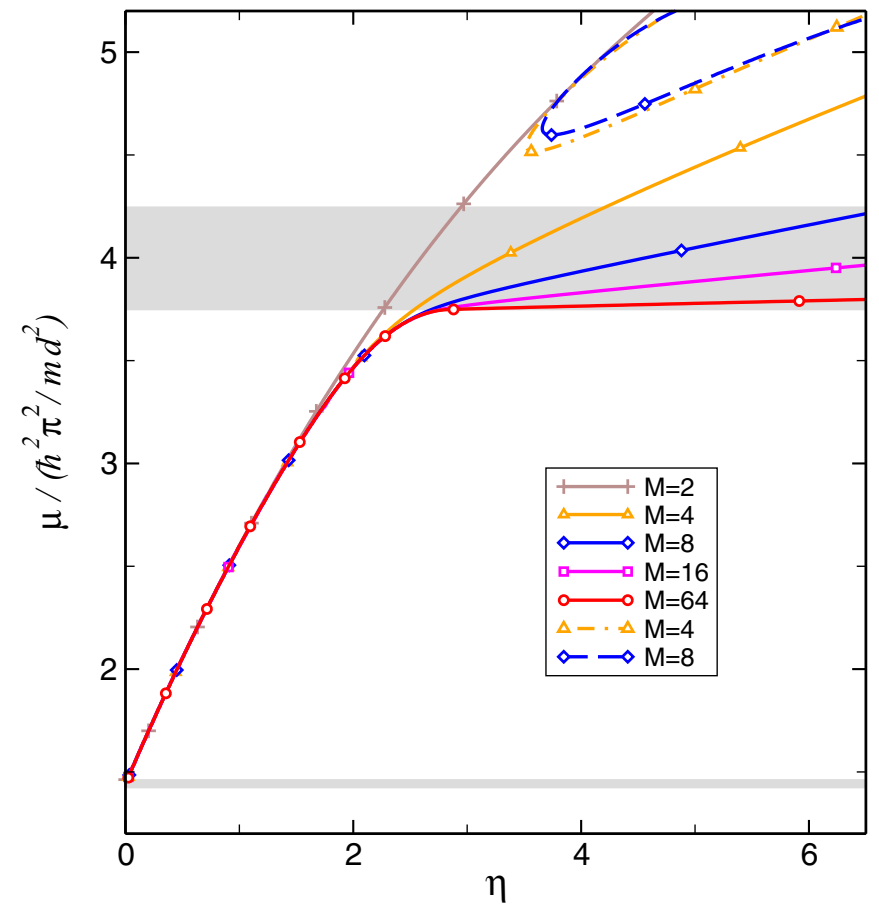

FIG. 9. Chemical potential versus interaction strength of gap solitons in ring lattices with varying number of sites $M$ but the same depth $s=10$ and spacing $d$. The main difference arises from the behavior at the resonance with the upper energy band. The phenomenology in high- $M$ lattices approaches the limit of infinite lattices, where gap solitons cannot live inside the bands. Saddle node bifurcations (dashed lines) appear in the second energy gap for M > 2 .

\section{Origin at the noninteracting regime}

As we have anticipated, the gap solitons bifurcate from the energy degeneracies of the linear regime. There, depending on the parity of the number of lattice sites $M$, the bifurcation takes place either at $\hat{\Omega} \neq 0$ when $M$ is even, or at $\hat{\Omega}=0$ when $M$ is odd.

For even $M$ there is just one (hence nondegenerate) linear Bloch state having $q=M / 2$ at the edge of the Brillouin zone. In this case, if one keeps $\hat{\Omega}=0$ the gap-soliton bifurcation is observed to emerge from the highest energy states of the nonlinear energy bands (see Figs. 3 and 11 for $M=4$ ), as usually reported. However, the gap-soliton families originate before, within the linear regime and at half-integer values of $\hat{\Omega}$, from the linear superposition of degenerate Bloch waves. In particular, the fundamental gap solitons (those localized in a single site, like states A and B in Fig. 11) arise from the highest energy degeneracy in the energy bands. The observed nonlinear bifurcation at $\hat{\Omega}=0$ occurs when the whole family of gap solitons forms a continuous energy band detached from the Bloch-wave families that were originally connected.

The described process is summarized in Fig. 10 for a minimal system with $M=2$ and $s=2$. The solid (dashed) lines correspond to a solitonic family originating from the real (complex) linear combination of degenerate Bloch waves at $\hat{\Omega}= \pm 0.5:|1,1\rangle_{r}=|1,1\rangle+|1,0\rangle$ and $|1,1\rangle_{i}=|1,1\rangle+$ $i|1,0\rangle$. These families, represented at different interaction
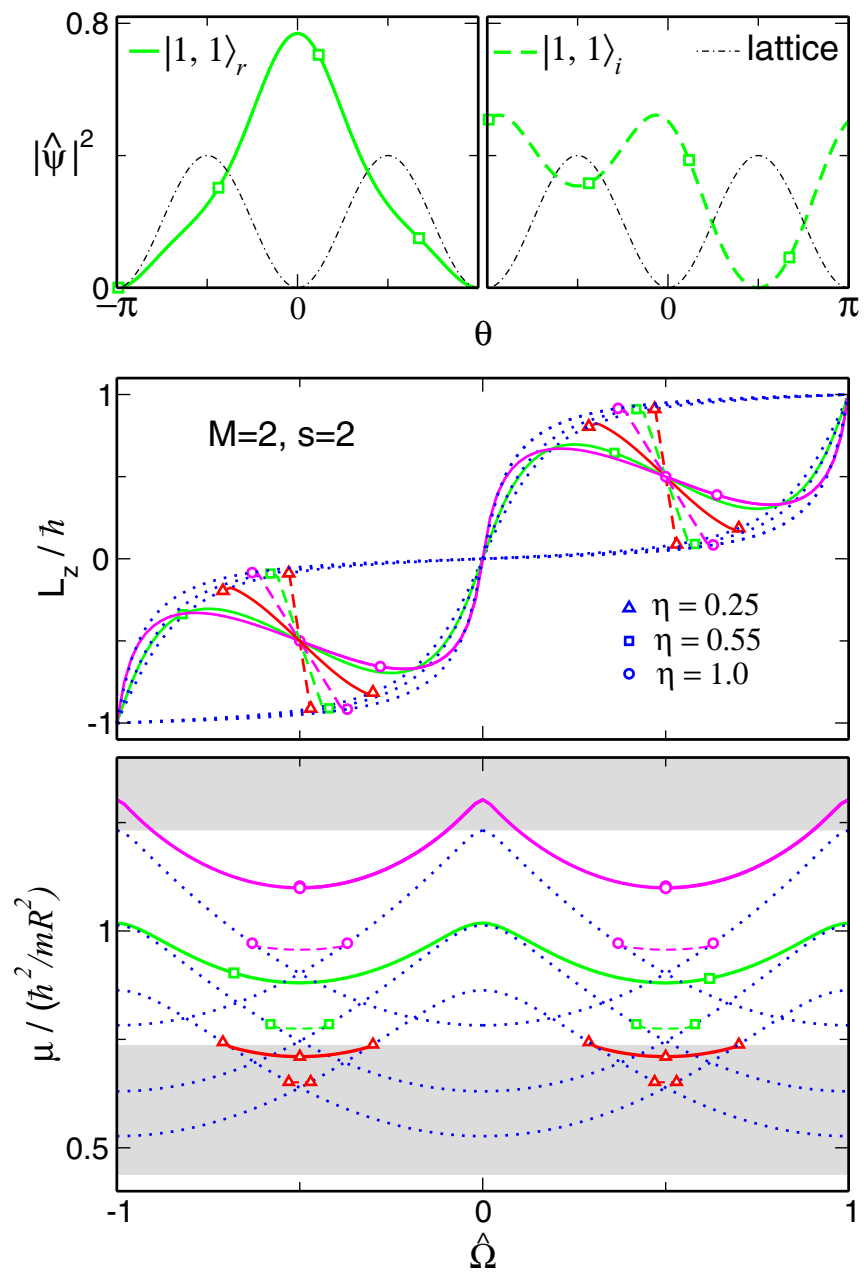

FIG. 10. Origin and evolution of gap solitons in a two-well rotating ring lattice with $s=2$ for varying interaction strength $\eta$. The two lower panels show the mean angular momentum and the chemical potential of the soliton families (solid and dashed lines) originating in the first energy band, where they connect the Blochwave states $|1,0\rangle$ and $|1,1\rangle$ (dotted lines). The top-row panels depict the gap-soliton density profiles (see text) at $\hat{\Omega}=0.5$ and $\eta=0.55$.

values in the two lower panels of Fig. 10, are made of states that show one and two density peaks in the ring, respectively (as can be seen in the top-row panels of Fig. 10). Their trajectories in the $\mu-\Omega$ graph shape swallowtail structures that are not qualitatively different from the intraband swallowtails previously associated with dark-soliton-like states.

The $\mu-\Omega$ graph in Fig. 10 also shows the detachment (at $\eta \approx 0.55$ ) of the families of fundamental gap solitons (solid curves) connecting two families of Bloch waves (dotted curves). Importantly, this detachment occurs inside the energy gap, and it is associated with the gap-soliton bifurcation observed at $\hat{\Omega}=0$. Equivalent processes take place for gap solitons emerging in higher energy gaps. This phenomenon can be put into correspondence with the metastable quantum phase transition described in Ref. [48] for states with attractive interaction in a ring without lattice. There, at a given interaction threshold, the bright-soliton families also separate from the winding number families that they connect, so that the 
solitons have always lower energy than the winding number states for given $\hat{\Omega}$. It is worth noticing that in the presence of the lattice, on the contrary, the bright-soliton-like states have higher energy than the Bloch states.

The interaction threshold for the detachment of the solitonic curves (or equivalently the gap-soliton bifurcation at $\hat{\Omega}=0$ ) varies with the radius of the ring and the depth of the lattice. Our numerical results show that the bifurcation point gets closer to the linear limit as the system approaches the infinite-lattice configuration. This fact can be qualitatively understood as a result of the changes produced in the energy bands, featured by a higher number of available states that are separated by lower energy increments. Under these conditions, the system can transfer such differences in kinetic energy of the extended states into interaction energy of a localized state at essentially the same value of the chemical potential. The same mechanism operates in deeper lattices, where there also exist a higher density of available states because of the reduced bandwidths.

When $M$ is odd the scenario is slightly different. There are two degenerate stationary states with definite quasimomentum at $\left|q_{M}\right|<M / 2$, which is the highest absolute value in the discrete band. At $\hat{\Omega}=0$, two independent linear combinations can be built from these states in the noninteracting regime, $\alpha\left|q_{M}\right\rangle+\beta\left|-q_{M}\right\rangle$, that show the typical features of gap solitons. Figures 3 (top panel) and 5 illustrate the origin and evolution of these gap solitons in the nonlinear regime for a lattice with $M=3$. The symmetric states, which are the nonlinear continuation of $|1,1\rangle_{s}=|1,1\rangle+|1,-1\rangle$, become more energetic than the antisymmetric ones, which are a continuation of $|1,1\rangle_{a}=|1,1\rangle-|1,-1\rangle$. Both families are made of real stationary states with zero mean angular momentum, and, unlike the states with definite $q$, present nonhomogeneous density profiles that break the symmetry of the lattice (as seen in the left panels of Fig. 6). For the interaction value of Fig. 5, both gap-soliton families form energy bands that are already detached from those of the Bloch-wave states.

The differences introduced by the parity of $M$ in the fewsite ring lattice are strongly reduced in long (high $M$ ) lattices. In the infinite-lattice limit the gap-soliton family bifurcates from the linear regime at $\hat{\Omega}=0$, although near the bifurcation point the states belonging to this branch cannot show a sharp localization. The longer the lattice the more advance within the nonlinear regime is needed in order to get a clear-cut density peak.

\section{Resonances with the linear energy bands}

We have summarized the configurations and bifurcations of bright soliton states in Fig. 11, inside a static ring lattice with $M=4$ sites and depth $s=10$. Within each energy gap a new family of bright solitons appears [54]; this is the case of solitons A and B inside the first and the second gap, respectively. These solitons present $n-1$ nodes ( $n$ being the lower band or gap index) inside the lattice site where they are localized. As we show below, these states can be traced back to the linear regime, where they originate in the interior of the energy band immediately below the gap where they first emerge. The other types of gap solitons ( $\mathrm{C}$ to I in Fig. 11) bifurcate
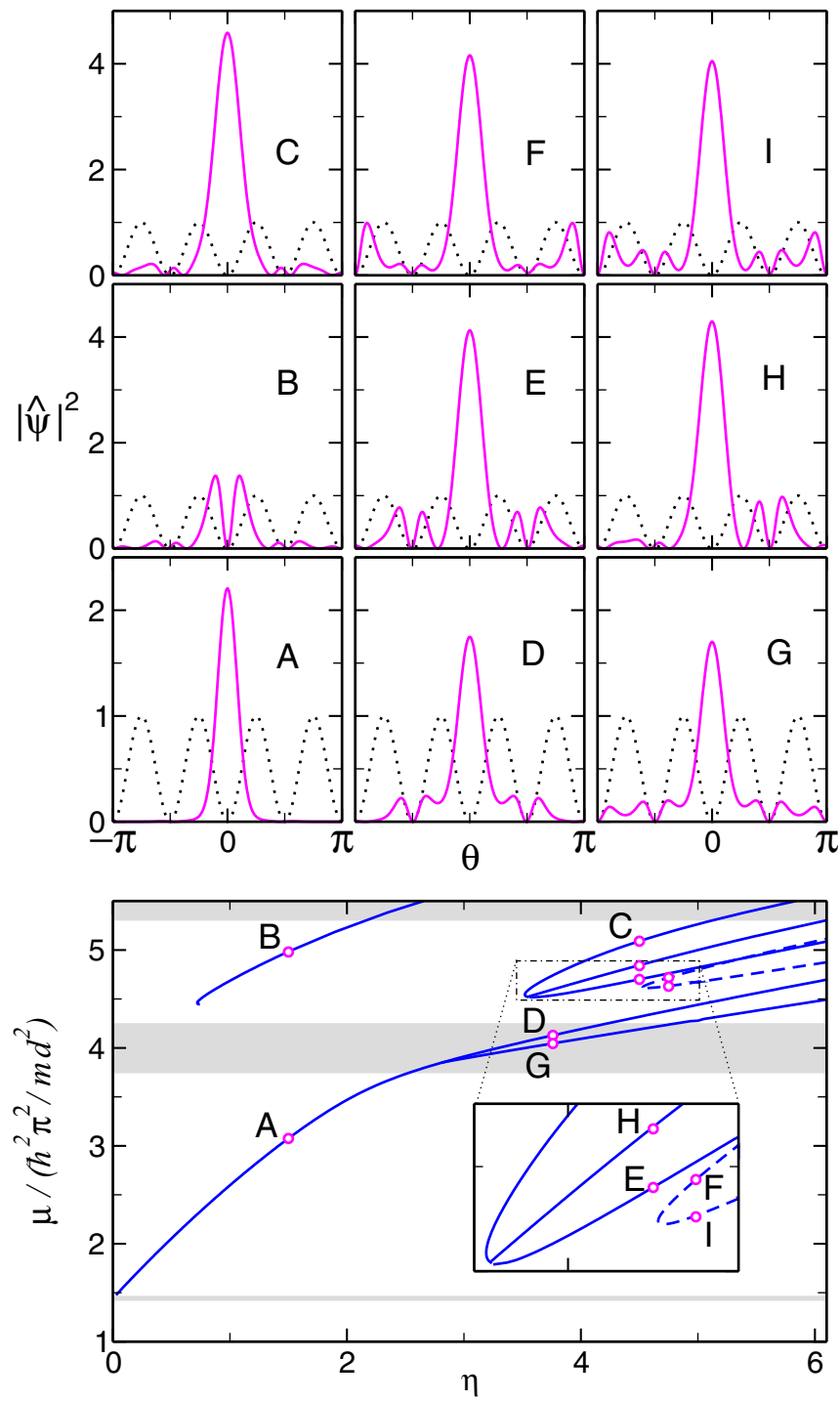

FIG. 11. Gap solitons in a ring lattice with $M=4, s=10$, and $\hat{\Omega}=0$. The bottom panel shows the trajectories of gap solitons in the $\mu-\eta$ graph over the underlying linear energy bands (gray-shadowed stripes). The upper panels labeled A-I depict the number density of the states (solid lines) marked by open symbols in the bottom panel; for reference, the lattice is represented by dotted lines.

from resonances with excitation modes proceeding from the linear Bloch waves. Among the bifurcations, the saddle node bifurcation is particularly relevant since it is responsible for the continuation of the soliton trajectories into upper energy gaps (like soliton C). The resonance of the bright soliton frequency with those of the linear spectrum is the key for the nonexistence of localized states inside the energy bands of infinite lattices [55]. In this regard, plausible arguments have been given in the realm of discrete systems [56]. Such resonances produce oscillating tails surrounding the soliton that have the structure of the resonant linear Bloch waves. The extended tails eventually dismantle the localized density peak. Practically the same happens in the ring lattice, with the peculiarity of the finite system to admit the coexistence of high-density peaks on the extended density tail. 


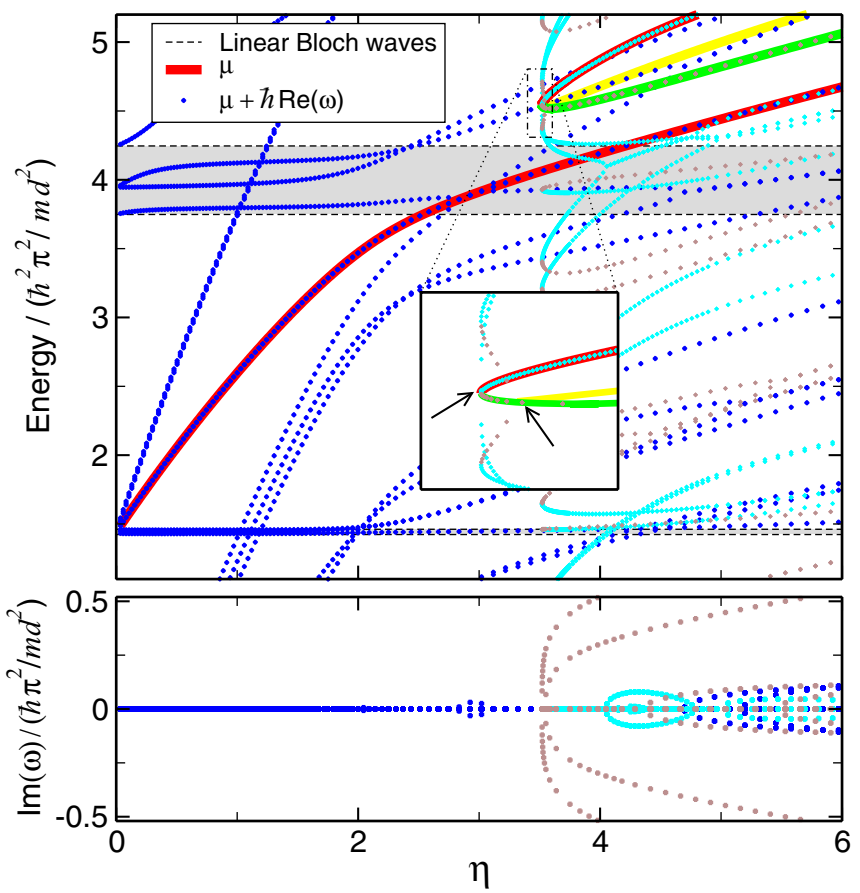

FIG. 12. Top panel: Chemical potential of fundamental gap solitons (thick lines) in the ring lattice of Fig. 11, and, superimposed, sum of chemical potential plus real part of Bogoliubov excitation energies (symbols) as a function of the interaction. The arrows in the inset point to the saddle node bifurcation (left arrow), due to the collision of gap solitons of type C and E in Fig. 11, and to a secondary bifurcation (right arrow) of solitons $\mathrm{E}$ to give rise to the family of states G. Bottom panel: Imaginary part of the frequencies of Bogoliubov excitations as a function of interaction.

Differently from the case of out-of-band swallowtails, gap solitons present linear excitations (proceeding from the top of the energy bands) that diverge from the underlying linear spectrum, along with excitation energies that keep a roughly constant value close (the longer the lattice the closer) to the linear spectrum (see Fig. 12). The collisions between constant and diverging excitation modes create a complex scenario of instability regions. Figure 12 illustrates this behavior for gap solitons in the static lattice with $M=4$ and $s=10$ of Fig. 11. Before (but close to) entering the energy band, the gap solitons in the first gap present instabilities (in small ranges of $\eta$ ) triggered by the collision at nonzero frequency $\hat{\omega}$ between the modes proceeding from the first and the third energy bands. When the gap solitons enter the energy band (like state D in Fig. 11), the appearance of an imaginary excitation frequency marks a pitchfork bifurcation of new families of soliton-like states (made of current-carrying states like G).

Within the second gap, a saddle node bifurcation is produced by the collision of two types of gap-soliton solutions with different symmetry ( $\mathrm{C}$ and $\mathrm{E}$ in Fig. 11). For this bifurcation to take place, it is necessary that $M>2$, since excitation modes with intermediate quasimomentum proceeding from the linear energy bands (other than those proceeding from the Brillouin zone edge) are needed to provide the specific density pattern in the neighbor sites of the soliton. Such modes are also allowing for alternative density patterns in
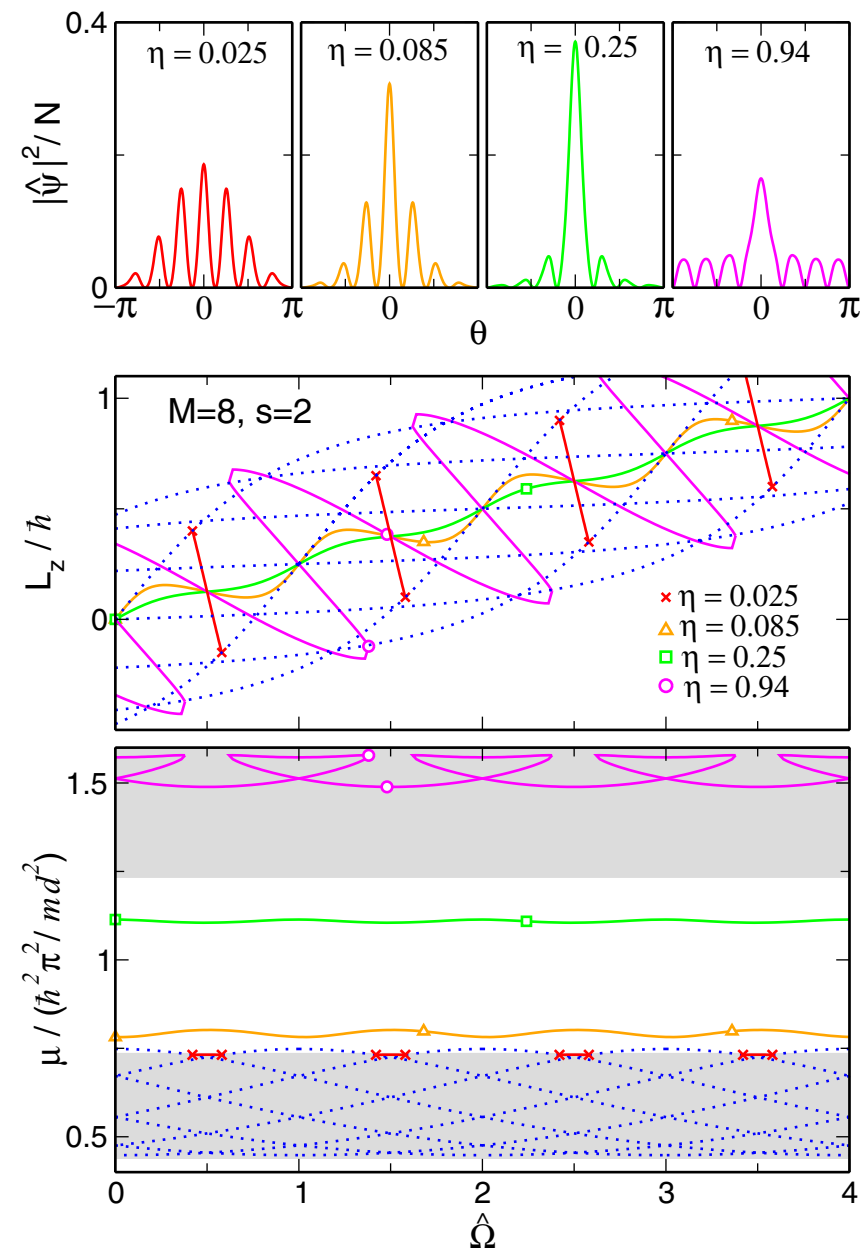

FIG. 13. Gap-soliton generation in an 8-site ring lattice with $s=$ 2 and varying interaction parameter $\eta=\hat{g} N / M$. The panels in the top row depict the soliton density at $\hat{\Omega}=0.5$ for increasing $\eta$. The two lower panels show the soliton angular momentum and chemical potential for varying rotation rate $\hat{\Omega}$. The dotted lines represent the nonlinear Bloch waves.

the soliton tail that produce secondary bifurcations (as the branch containing state $\mathrm{H}$ ). Furthermore, additional saddle node bifurcations involving current states (like F and I) take place at higher interaction. For increasing $M$, there is also an increasing number of density configurations available around the soliton peaks, and therefore longer lattices entail larger sets of solitonic states.

\section{Gap solitons in longer lattices}

In order to see the correspondence with longer lattices, we have chosen a system with $M=8$ and equal lattice spacing as the minimal system of Fig. 10. In Fig. 13, one can see that the mechanism for the generation of bright solitons is the same. They emerge from the linear regime and from the connection between Bloch waves. The separation of the chemical potential curve takes place within the energy gaps for interaction energies that are lower than those found in the shorter lattice. In addition, the soliton energy band becomes flatter far from the first (linear) energy band, showing particlelike features, i.e., flat energy in the comoving reference frame, 
and linear increase in momentum. For higher nonlinearity, the resonance with the second energy band increases the soliton bandwidth and leads to a bifurcation with the generation of swallowtails similar to those developed by nonlinear Bloch waves.

\section{CONCLUSIONS}

The small ring lattices are minimal systems that can be mapped to the infinite lattice by the introduction of angular rotation. In this way, and due to the existence of a small number of available states, the ring lattices allow for a simpler analysis of the states of equilibrium. In particular, we have addressed the origin, range of existence, and stability of solitonic states. Our results show that they share a general common origin with the nonlinear Bloch waves that can be tracked up to the linear regime. While the Bloch waves are states with definite quasimomentum that follow the symmetry of the underlying lattice, the solitonic states emerge as a linear combination of Bloch waves and break this symmetry. The symmetrybreaking states have both a nodal structure as well as a local density maximum and could thus be classified as either dark or bright solitons. Clear-cut features are only shown in the dark-soliton states of the out-of-band swallowtails, or in the (fundamental) gap-soliton states that bifurcate from the top of the energy bands.

The resonances of the nonlinear waves with the underlying linear spectrum produce dynamical instabilities and new bifurcations. Among them, we have shown how the pitchfork bifurcation of the highest-energy nonlinear Bloch waves give rise to out-of-band swallowtails, how the saddle node bifurcation of fundamental gap solitons continues the soliton families into higher energy gaps, and how the ring lattice provides a plausible demonstration of the nonexistence of localized states inside the linear energy bands of the infinite lattice.

The small ring lattices could play an interesting role in BEC experiments in order to facilitate the materialization of controlled persistent currents or even solitonic states. Previous experiments have made use of a Gaussian-shaped barrier potential to drag a toroidal system into a persistent current state [45], but this procedure involves sudden phase slips that produce a transitory unstable stage. The present work shows that a periodic potential can provide a simpler and adiabatic way to transit between quantized persistent currents. The experimental realization of BECs loaded on $1 \mathrm{D}$ rotating ring lattices is currently feasible, for instance, by means of painted optical-dipole potentials [17]. With this technique, a laser sheet keeps the atomic cloud confined to a $2 \mathrm{D}$ plane, on the top of which a fast-scanning (typically 4-5 kHz) laser beam can build a time-averaged atomic-ring trap with periodic, even rotating, geometry [17]. For example, for ${ }^{87} \mathrm{Rb}$ atomic BECs, a ring lattice with $M=4$ sites could be realized using $\lambda=$ $1064 \mathrm{~nm}$ lasers with axial beams of $2.5 \mu \mathrm{m}$ waist shining a perpendicular sheet of $7.5 \mu \mathrm{m}$ waist. A ring radius of $R=$ $20 \mu \mathrm{m}$ would give $\Omega_{R}=1.8 \mathrm{~Hz}$, and provide the system with an effective 1D geometry of large (circumference to transverse width) aspect ratio. With this arrangement, a transit up to a rotation rate $\hat{\Omega}=4$ (that is, $\Omega=7.3 \mathrm{~Hz}$, a feasible timeaveraged dynamic potential) could lead the system into a state with $L_{z}=4 \hbar$. The realization of solitonic states seems more challenging, however, because the lattice symmetry has to be broken by the atomic cloud. This could be achieved by the combination of a precise rotation rate and a symmetry-broken initial state in analogy to the experiment of Ref. [4], where gap solitons were produced in a linear lattice.

\section{ACKNOWLEDGMENTS}

A.M.M. is grateful to Sergej Flach for insightful discussions. V.D. acknowledges financial support from Ministerio de Economía y Competitividad (Spain) and Fondo Europeo de Desarrollo Regional (FEDER, EU) under Grants No. FIS2013-41532-P and No. FIS2016-79596-P. M.G. and R.M. acknowledge financial support from Ministerio de Economía y Competitividad (Spain) and Fondo Europeo de Desarrollo Regional (FEDER, EU) under Grants No. FIS2014-52285C2-1-P and No. FIS2017-87801-P. J.B. acknowledges funding by the Marsden Fund of New Zealand (Contract No. MAU1604), from government funding managed by the Royal Society Te Apārangi.
[1] I. Bloch, J. Dalibard, and S. Nascimbène, Nat. Phys. 8, 267 (2012).

[2] O. Morsch and M. Oberthaler, Rev. Mod. Phys. 78, 179 (2006).

[3] M. Ben Dahan, E. Peik, J. Reichel, Y. Castin, and C. Salomon, Phys. Rev. Lett. 76, 4508 (1996).

[4] B. Eiermann, T. Anker, M. Albiez, M. Taglieber, P. Treutlein, K.-P. Marzlin, and M. K. Oberthaler, Phys. Rev. Lett. 92, 230401 (2004).

[5] L. F. Mollenauer and J. P. Gordon, Solitons in Optical Fibers: Fundamentals and Applications (Academic Press, Boston, 2006).

[6] S. Burger, F. S. Cataliotti, C. Fort, F. Minardi, M. Inguscio, M. L. Chiofalo, and M. P. Tosi, Phys. Rev. Lett. 86, 4447 (2001).

[7] M. Greiner, I. Bloch, O. Mandel, T. Hänsch, and T. Esslinger, Appl. Phys. B 73, 769 (2001).

[8] M. Greiner, O. Mandel, T. Esslinger, T. W. Hänsch, and I. Bloch, Nature (London) 415, 39 (2002).
[9] P. J. Y. Louis, E. A. Ostrovskaya, C. M. Savage, and Y. S. Kivshar, Phys. Rev. A 67, 013602 (2003).

[10] A. M. Mateo, V. Delgado, and B. A. Malomed, Phys. Rev. A 83, 053610 (2011).

[11] A. M. Mateo and V. Delgado, J. Phys. A: Math. Theor. 47, 245202 (2014).

[12] B. Wu and Q. Niu, Phys. Rev. A 64, 061603 (2001).

[13] B. Wu and Q. Niu, New J. Phys. 5, 104 (2003).

[14] M. Krämer, C. Menotti, L. Pitaevskii, and S. Stringari, Eur. Phys. J. D 27, 247 (2003).

[15] H. Sakaguchi and B. A. Malomed, Phys. Rev. A 79, 043606 (2009).

[16] S. Franke-Arnold, J. Leach, M. J. Padgett, V. E. Lembessis, D. Ellinas, A. J. Wright, J. M. Girkin, P. Öhberg, and A. S. Arnold, Opt. Express 15, 8619 (2007).

[17] K. Henderson, C. Ryu, C. MacCormick, and M. Boshier, New J. Phys. 11, 043030 (2009). 
[18] G. Gauthier, I. Lenton, N. M. Parry, M. Baker, M. Davis, H. Rubinsztein-Dunlop, and T. Neely, Optica 3, 1136 (2016).

[19] C. Kittel, Introduction to Solid State Physics (Wiley, New York, 1996).

[20] L. Amico, D. Aghamalyan, F. Auksztol, H. Crepaz, R. Dumke, and L. C. Kwek, Sci. Rep. 4, 04298 (2014).

[21] D. Aghamalyan, M. Cominotti, M. Rizzi, D. Rossini, F. Hekking, A. Minguzzi, L.-C. Kwek, and L. Amico, New J. Phys. 17, 045023 (2015).

[22] M. Koláŕ, T. Opatrný, and K. K. Das, Phys. Rev. A 92, 043630 (2015).

[23] G. Arwas, A. Vardi, and D. Cohen, Sci. Rep. 5, 13433 (2015).

[24] A. Gallemí, M. Guilleumas, J. Martorell, R. Mayol, A. Polls, and B. Juliá-Díaz, New J. Phys. 17, 073014 (2015).

[25] A. Gallemí, M. Guilleumas, J. Martorell, R. Mayol, A. Polls, and B. Juliá-Díaz, New J. Phys. 18, 075005 (2016).

[26] H. M. Cataldo and D. M. Jezek, Phys. Rev. A 84, 013602 (2011).

[27] G. S. Paraoanu, Phys. Rev. A 67, 023607 (2003).

[28] B. Wu and Q. Niu, Phys. Rev. A 61, 023402 (2000).

[29] J. C. Bronski, L. D. Carr, B. Deconinck, and J. N. Kutz, Phys. Rev. Lett. 86, 1402 (2001).

[30] D. Diakonov, L. M. Jensen, C. J. Pethick, and H. Smith, Phys. Rev. A 66, 013604 (2002).

[31] M. Machholm, C. J. Pethick, and H. Smith, Phys. Rev. A 67, 053613 (2003).

[32] E. J. Mueller, Phys. Rev. A 66, 063603 (2002).

[33] C. Ryu, M. F. Andersen, P. Cladé, V. Natarajan, K. Helmerson, and W. D. Phillips, Phys. Rev. Lett. 99, 260401 (2007).

[34] S. Moulder, S. Beattie, R. P. Smith, N. Tammuz, and Z. Hadzibabic, Phys. Rev. A 86, 013629 (2012).

[35] A. C. Bleszynski-Jayich, W. E. Shanks, B. Peaudecerf, E. Ginossar, F. von Oppen, L. Glazman, and J. G. E. Harris, Science 326, 272 (2009).

[36] H. Bluhm, N. C. Koshnick, J. A. Bert, M. E. Huber, and K. A. Moler, Phys. Rev. Lett. 102, 136802 (2009).

[37] J. D. Reppy and D. Depatie, Phys. Rev. Lett. 12, 187 (1964).
[38] A. Ramanathan, K. C. Wright, S. R. Muniz, M. Zelan, W. T. Hill, C. J. Lobb, K. Helmerson, W. D. Phillips, and G. K. Campbell, Phys. Rev. Lett. 106, 130401 (2011).

[39] S. Beattie, S. Moulder, R. J. Fletcher, and Z. Hadzibabic, Phys. Rev. Lett. 110, 025301 (2013).

[40] A. Y. Cherny, J.-S. Caux, and J. Brand, Front. Phys. 7, 54 (2012).

[41] B. S. Deaver and W. M. Fairbank, Phys. Rev. Lett. 7, 43 (1961).

[42] K. A. Matveev, A. I. Larkin, and L. I. Glazman, Phys. Rev. Lett. 89, 096802 (2002).

[43] L. Amico, A. Osterloh, and F. Cataliotti, Phys. Rev. Lett. 95, 063201 (2005).

[44] M. Cominotti, D. Rossini, M. Rizzi, F. Hekking, and A. Minguzzi, Phys. Rev. Lett. 113, 025301 (2014).

[45] S. Eckel, J. G. Lee, F. Jendrzejewski, N. Murray, C. W. Clark, C. J. Lobb, W. D. Phillips, M. Edwards, and G. K. Campbell, Nature (London) 506, 200 (2014).

[46] A. Muñoz Mateo, A. Gallemí, M. Guilleumas, and R. Mayol, Phys. Rev. A 91, 063625 (2015).

[47] Y. V. Kartashov, B. A. Malomed, V. A. Vysloukh, and L. Torner, Opt. Lett. 33, 2949 (2008).

[48] R. Kanamoto, L. D. Carr, and M. Ueda, Phys. Rev. A 79, 063616 (2009).

[49] O. Fialko, M.-C. Delattre, J. Brand, and A. R. Kolovsky, Phys. Rev. Lett. 108, 250402 (2012).

[50] Y. Li, W. Pang, and B. A. Malomed, Phys. Rev. A 86, 023832 (2012).

[51] L. Pitaevskii and S. Stringari, Bose-Einstein Condensation and Superfluidity (Oxford University Press, Oxford, 2016).

[52] A. Barone and G. Paterno, Physics and Applications of the Josephson Effect (Wiley, New York, 1982).

[53] M. Machholm, A. Nicolin, C. J. Pethick, and H. Smith, Phys. Rev. A: At., Mol., Opt. Phys. 69, 043604 (2004).

[54] Y. Zhang, Z. Liang, and B. Wu, Phys. Rev. A 80, 063815 (2009).

[55] V. Delgado and A. M. Mateo, Sci. Rep. 8, 10940 (2018).

[56] S. Flach and C. R. Willis, Phys. Rep. 295, 181 (1998). 